\title{
Bayesian Cox Proportional Hazards Model in Survival Analysis of HACE1 Gene with Age at Onset of Alzheimer's Disease
}

\author{
Ke-Sheng Wang ${ }^{1}$, Ying Liu', Shaoqing Gong ${ }^{2}$, Chun $X u^{3}$, Xin Xie ${ }^{4}$, Liang Wang ${ }^{1}$ and Xingguang Luo ${ }^{5,6}$
}

${ }^{1}$ Department of Biostatistics and Epidemiology, College of Public Health, East Tennessee State University, Johnson City, TN, USA

${ }^{2}$ School of Public Policy and Administration, Xi'an Jiaotong University, Xi'an, China

${ }^{3}$ Department of Health and Biomedical Sciences, College of Health Affairs, University of Texas Rio Grande Valley, Brownsville, TX, USA

${ }^{4}$ Department of Economics and Finance, College of Business and Technology, East Tennessee State University, Johnson City, TN, USA

${ }^{5}$ Department of Psychiatry, Yale University School of Medicine, New Haven, CT, USA

${ }^{6}$ Biological Psychiatry Research Center, Beijing Huilongguan Hospital, Beijing, China

*Corresponding author: Ke-Sheng Wang, Department of Biostatistics and Epidemiology, College of Public Health, East Tennessee State University, PO Box 70259, Lamb Hall, Johnson City, TN 37614-1700, USA; Tel: +1-423-439-4481; Fax: +1 423-439-4606; E-mail: wangk@etsu.edu

\begin{abstract}
Alzheimer's Disease (AD), the most common form of dementia, is a chronic neurodegenerative disease. The HECT domain and ankyrin repeat containing E3 ubiquitin protein ligase 1 (HACE1) gene is expressed in human brain and may play a role in the pathogenesis of neurodegenerative disorders. Till now, no previous study has reported the association of the HACE1 gene with the risk and Age at Onset (AAO) of $A D$; while few studies have checked the proportional hazards assumption in the survival analysis of AAO of $A D$ using Cox proportional hazards model. In this study, we examined the associations of 14 Single Nucleotide Polymorphisms (SNPs) in the HACE1 gene with the risk and the $A A O$ of $A D$ using $791 A D$ patients and 782 controls. Multiple logistic regression model identified one SNP (rs9499937 with $p=1.8 \times 10^{-3}$ ) to be associated with the risk of AD. For survival analysis of AAO, both classic Cox regression model and Bayesian survival analysis using the Cox proportional hazards model were applied to examine the association of each SNP with the AAO. The Hazards Ratio (HR) with its 95\% Confidence Interval $(\mathrm{Cl})$ was estimated. Survival analysis using the classic Cox regression model showed that 4 SNPs were significantly associated with the AAO (top SNP rs9499937 with $\mathrm{HR}=1.33,95 \% \mathrm{Cl}=1.13-1.57, \mathrm{p}=5.0 \times$ $10^{-4}$ ). Bayesian Cox regression model showed similar but a
\end{abstract}

slightly stronger associations (top SNP rs9499937 with HR $=1.34,95 \% \mathrm{Cl}=1.11-1.55$ ) compared with the classic Cox regression model. Using an independent family-based sample, one SNP rs9486018 was associated with the risk of $A D(p=0.0323)$ and the T-T-G haplotype from rs9786015, rs9486018 and rs4079063 showed associations with both the risk and AAO of AD ( $p=2.27 \times 10^{-3}$ and 0.0487 , respectively). The findings of this study provide first evidence that several genetic variants in the HACE1 gene were associated with the risk and $A A O$ of $A D$.

\section{Keywords}

Alzheimer's disease, Age at onset, HACE1, Single nucleotide polymorphisms, Survival analysis, Cox model, Bayesian analysis

\section{Introduction}

Alzheimer's Disease (AD) is a chronic neurodegenerative disease [1]. It is estimated that, in 2010, about 4.7 million people in the United States (US) aged 65 years or older live with $A D$, and this number is projected to rise to 13.8 million, a nearly three-fold increase, by 2050 [2]. Weuve et al. [3] estimated the number of adults (aged 
$\geq 65$ years) with $A D$ in each US state and the District of Columbia (DC) and found that the number of older adults (aged $\geq 65$ years) with $A D$ in the US ranged from 5.1 (Alaska) to 530 million (California) in 2010; while in 2010 , older adults with AD dementia comprised a median of $1.6 \%$ of a given state's total population; whereas by 2025 , throughout the US, this proportion will keep increasing by nearly one-third on average [3]. The prevalence was estimated to be about $3.12 \%$ in 2012 for older adults ( $\geq 60$ years) in the US using the Nationwide Inpatient Sample (NIS) 2002-2012 [4]. A meta-analysis estimated the prevalence for the combined population of African-Americans and Caucasians aged 65-90 in 2013 was $5.7 \%$ in the US; the prevalence for African-Americans aged $65-90$ years was $8.6 \%$, compared to $5.5 \%$ for Caucasians [5]. Globally, 26.6 million people $(0.40 \%$ of the world population) suffered from AD in 2006 and it was predicted to affect 1 in 85 people by 2050 [6]. A recent meta-analysis showed that the prevalence of $A D$ in Europe was $5.05 \%$ for adult (aged $\geq 50$ years) $(3.31 \%$ for males and $7.13 \%$ females) [7]. Increasing evidence suggests that autophagy may play a central role in $A D$ $[8,9]$. The genetic heritability of $A D$ ranges from $49 \%$ to $79 \%$ based on reviews of twin and family studies [10]. In addition to the risk, the Age at Onset (AAO) of AD has a genetic component with heritability about $42 \%[11,12]$.

The HECT domain and ankyrin repeat containing E3 ubiquitin protein ligase 1 (HACE1) gene (also known as KIAA1320) is located at 6q16.3 [13-15]. The HACE1 is expressed in brain, heart, lung, kidney, testis, and ovary $[13,15]$. Several studies have implicated that HACE1 is a candidate chromosome $6 \mathrm{q} 21$ tumor suppressor gene involved in multiple cancers [16-18]. Recently, it has been reported that HACE1 gene may play a role in neurodegeneration [19] and autophagy pathway [20]; while HACE1 mutations are involved in an autosomal recessive neurodevelopmental disorder [21], and glutamine addiction [22]. A Genome-Wide Association Study (GWAS) identified five Single-Nucleotide Polymorphisms (SNPs) (rs17065302, rs11759010, rs6927608, rs4946645 and rs4245525) within the HACE1 gene associated with equol-producing phenotype such as blood pressure, which may implicate HACE1 in intestinal immune responses [23]. Another GWAS identified 5 SNPs (rs4336470. rs9404576, rs4079063, rs24996663, and rs2499667) in HACE1 associated with neuroblastoma susceptibility [24]; while in a replication study, rs4336470 showed moderate association $(p<0.05)$ with risk of neuroblastoma [25]. More recently, it has been reported that the five above SNPs in the HACE1 gene may have a weak combined effect $(p=0.065)$ on neuroblastoma risk in Southern Chinese children [26]. Thus we hypothesized that HACE1 genetic variants may be in association with $A D$ development.

To our best knowledge, no study has focused on the association of the HACE1 gene with the risk and AAO of AD. Even though the Cox proportional hazards model has been used to detect genetic associations with the $A A O$ of $A D$ [27-29], the proportional hazards assumption may be violated and they might not be carefully checked. Bayesian methods have been widely used recently in genetic association studies and provide alternative ways to traditional statistical methods [30-32]. In this study, we explored the association of HACE1 with the $A A O$ of $A D$ by using a Bayesian proportional hazards model in a population-based sample and then a family-based sample for replication.

\section{Subjects and Methods}

\section{Study population}

791 patients with AD and 782 controls with complete genotype and phenotype information in a Canadian sample were selected from the Multi-Site Collaborative Study for Genotype-Phenotype Associations in Alzheimer's disease and the longitudinal follow-up of Genotype-Phenotype Associations in Alzheimer's disease and the Neuroimaging component of Genotype-Phenotype Associations in Alzheimer's disease-Study Accession: phs000219.v1.p1. Covariates include sex and age. The details about these subjects were described in previous studies $[27,33]$. Genotyping was conducted using the Affymetrix technique. The genotypes of 14 SNPs within the HACE1 gene were available in this data.

A family-based study (1266 AD cases and their relatives, 1070 individuals with the AAO values) were available from the National Institute on Aging - Late Onset Alzheimer's Disease (NIA-LOAD) Family Study: Genome-Wide Association Study for Susceptibility Loci - Study Accession: phs000168.v1.p1. Genotyping by the Center for Inherited Disease Research (CIDR) was performed using the Illumina Infinium II assay protocol. The details about the sample of subjects were described elsewhere [34]. There are 28 SNPs within the HACE1 gene in this family-based sample.

\section{Statistical analysis}

Descriptive statistics and genotype quality control: Descriptive statistics were used to characterize participants' sex, age and the AAO of AD stratified by AD case and control status [29,35]. Hardy-Weinberg Equilibrium (HWE) was tested for all SNPs using the controls by HAPLOVIEW software [36]. Then, Minor Allele Frequency (MAF) was determined for each SNP. Pair wise Linkage Disequilibrium (LD) statistics $\left(r^{2}\right)$ among SNPs were assessed using the European sample from the HapMap dataset (http://hapmap.ncbi.nlm.nih.gov/) and the founders in the family study.

Multiple logistic and linear regression models in PLINK software: Multiple logistic regression analysis of each SNP with the risk of $A D$ as a binary outcome, adjusted for sex and age, was performed using PLINK [37]; while the asymptotic $p$-values were obtained and the Odds Ratio (OR) and 95\% Confident Interval (Cl) were 
estimated. The parallel procedure was performed for the multiple linear regression analysis of each SNP with the $A A O$ of $A D$ as a continuous outcome. Bonferroni correction $\left(\alpha=0.05 / 14=3.57 \times 10^{-3}\right)$ was used to deal with the multiple comparison issue [38].

Bayesian Cox proportional hazards model in PROC PHREG: The Cox proportional hazards model (1) or Cox regression model [39] is widely used in the analysis of time-to-event data [40-42].

$$
h(t \mid x)=h_{0}(t) \exp \left(\beta_{1} S N P_{k}+\beta_{2} \operatorname{Sex}+\beta_{3} \text { Age }\right)
$$

where $h(t / x)$ is the hazard at time $\mathrm{t}$ for a subject (AAO for this study), $h_{0}(t)$ is the baseline hazard function. The Hazard Ratio (HR) is defined as the ratio of the predicated hazard function under two different values of a predictor variable. The PHREG procedure in SAS fits the Cox model by maximizing the partial likelihood function. Both the graphical and numerical methods [43] were used to check the proportional hazards assumption in the ASSESS option of PROC PHREG. The ASSESS option plots the cumulative score residuals against time for each independent variable; while the RESAMPLE option computes the $p$-value of a Kolmogorov-type supremum test based on a sample of 1,000 simulated residual patterns. A significant $p$-value indicates a poor fit.

The Akaike Information Criterion (AIC) (2) was used as a measure of better fit among candidate models $[44,45]$.

$$
\mathrm{AIC}=-2 \ln \{p(x \mid \hat{\theta})\}+2 k
$$

where $x$ is the random variable, $\hat{\theta}$ is the maximum likelihood estimate, and $k$ is the number of parameters. A smaller AIC generally indicates a better fit.

Bayesian statistics is an extension of Bayes theorem, which can be written as ( 3 )

$$
P(\theta \mid Y)=\frac{P(Y \mid \theta) P(\theta)}{P(Y)}
$$

where $\theta$ is the parameter of interest, $Y$ is the observed evidence, $P(Y)$ is the marginal probability, $P(Y \mid \theta)$ is the likelihood function, $P(\theta)$ is the prior, and $P(\theta / Y)$ is the posterior probability [32]. Bayesian Cox regression can be requested by using the BAYES statement in the PHREG procedure. Summary statistics (Mean, Standard Deviation, the Highest Posterior Density (HPD) and Credible Intervals, and Correlation Matrix) were com- puted for each of the parameters. Trace plots, posterior density plots, and autocorrelation function plots were also provided [32]. For Bayesian survival analysis of the $A A O$ of $A D$, the normal prior was chosen for the coefficients and the Deviance Information Criteria (DIC) was available instead of AIC. DIC is intended as a generalization of AIC [46]. A measure of effective numbers of parameters is defined as $p_{D}$ in (4), where $p_{D}$ is the posterior mean deviance minus the deviance measured at the posterior mean of the parameters.

$$
p_{D}=E[-2 \ln \{p(x \mid \theta)\}]+2 \ln \{p(x \mid \hat{\theta})\}
$$

Then DIC is defined analagously to AIC as in (5). Models with smaller DIC are better supported by the data.

$$
\mathrm{DIC}=-2 \ln \{p(x \mid \hat{\theta})\}+p_{\mathrm{D}}
$$

The PHREG procedure in SAS was used to fit the Cox model. Multiple Cox regression model analysis, adjusted for sex and age, was conducted to examine association of each SNP with the AAO of AD. Descriptive statistics and Cox regression analysis were performed with SAS v.9.4 (SAS Institute, Cary, NC, USA).

Family-based study: A family-based association analysis for $A D$ was performed using PBAT version 3.6.7 [47]. For the risk of $A D$, the Family-Based Association Test using Generalized Estimating Equations (FBAT-GEE) was used [48]; while for the AAO, FBAT-Wilcoxon statistics were employed [49]. The AAO values for healthy siblings were censored and age at entry into the study was used. Haplotype analysis was conducted in 2 or 3-SNP sliding window.

\section{Results}

\section{Descriptive statistics and genotype quality control}

The demographic characteristics of the subjects are detailed in Table 1. The mean AAO for cases was 76.4 and 72.3 years, respectively, in the NIA and Canadian samples, respectively. All 14 SNPs had MAF $>5 \%$ and were in HWE in the controls $(p>0.05)$; while 1 of 28 SNPs with HWE $<0.10^{-4}$ in the family sample was removed for further analysis.

\section{Multiple linear and logistic regression analyses using PLINK}

We found that one SNP was associated with the risk of $A D$ ( $r s 9499937$ with $\left.p=1.8 \times 10^{-3}\right)$ and four SNPs were

Table 1: Descriptive characteristics of cases and controls.

\begin{tabular}{|l|l|l|l|l|}
\hline Variable & \multicolumn{2}{l|}{ Family study (NIA sample) } & \multicolumn{2}{l|}{ Case-control study (Canadian sample) } \\
\hline & Patients & Controls & Patients & Controls \\
\hline Sample size $(\mathrm{n})$ & 1266 & 1279 & 791 & 782 \\
\hline Mean of age at onset (years \pm SD) & $76.4( \pm 6.7)$ & - & $72.3( \pm 8.5)$ & - \\
\hline Median of age at onset (years) & 77 & - & 73 & - \\
\hline Range of age at onset (years) & $50-98$ & - & $40-97$ & - \\
\hline Mean age at entry (years \pm SD) & - & $75.5( \pm 8.1)$ & $77.6( \pm 8.6)$ & $73.4( \pm 7.9)$ \\
\hline Median age at entry (years) & - & 75 & 79 & 79 \\
\hline Range of age at entry (years) & - & $42-103$ & $43-100$ & $48-94$ \\
\hline
\end{tabular}


associated with the AAO of AD (rs7746856, rs6941988, rs9499937 and rs7770002 with $p=3.09 \times 10^{-2}, 3.88 \times$ $10^{-3}, 7.39 \times 10^{-4}$, and $3.14 \times 10^{-2}$, respectively) (Table 2 ). Interestingly, the same SNP rs9499937 showed associations with both the risk and $A A O$ of $A D$ and the results remained significant after Bonferroni correction $(p<$ $\left.3.57 \times 10^{-3}\right)$.

\section{Supremum test for proportional hazards assumption}

Figure 1 and Figure 2 display the observed standardized score process with 20 simulated realizations from the null distribution for rs9499937 CC and CT genotypes, respectively. The plots showed that the observed process was atypical compared to the simulated realizations and revealed proportional hazards for the two genotypes compared with TT. The Kolmogorov-type su- premum test results based on 1,000 simulations for all the covariates were not significant $(p>0.05)$, suggesting the proportional hazards assumption was valid for all the variables in the Canadian sample.

\section{Classic Bayesian Cox proportional hazards model us- ing PROC PHREG}

The classic Cox model showed that four SNPs were associated with the AAO of AD (the CT genotype of the top SNP rs9499937 with $\mathrm{HR}=1.33,95 \% \mathrm{Cl}=1.13-1.57$, $\left.p=5.0 \times 10^{-4}\right)$. The HRs based on the Bayesian survival analyses revealed similar but a slightly stronger associations compared with the non-Bayesian analyses results (Table 3). The DIC for the four SNPs were similar to those of AIC. The trace plot, posterior density plot, and autocorrelation function plot based on Bayesian

Table 2: SNPs associated with the risk and/or age at onset of $A D(p<0.05)$.

\begin{tabular}{|l|l|l|l|l|l|l|l|l|}
\hline SNP & Position & Allele $^{\mathbf{a}}$ & MAF $^{\mathbf{b}}$ & HWE $^{\mathbf{c}}$ & OR-AD $^{\mathbf{d}}$ & p-AD $^{\mathbf{e}}$ & $\boldsymbol{\beta}_{-A A O^{\mathbf{f}}}$ & p-AAO $^{\mathbf{g}}$ \\
\hline rs7746856 & 105253053 & A & 0.47 & 0.787 & $0.92(0.79-1.06)$ & 0.246 & $-0.367(-0.70,-0.034)$ & 0.0309 \\
\hline rs6941988 & 105253349 & C & 0.47 & 0.84 & $0.92(0.79-1.06)$ & 0.239 & $-0.351(-0.68,-0.019)$ & 0.00388 \\
\hline rs9499937 & 105273953 & T & 0.19 & 0.649 & $0.73(0.60-0.89)$ & $1.80 \times 10^{-3}$ & $-0.79(-1.25,-0.34)$ & $7.39 \times 10^{-4}$ \\
\hline rs7770002 & 105280138 & A & 0.47 & 0.96 & $0.92(0.80-1.07)$ & 0.268 & $-0.366(-0.70,-0.033)$ & 0.0314 \\
\hline
\end{tabular}

aMinor allele; ${ }^{b}$ Minor allele frequency; ${ }^{c}$ Hardy-Weinberg equilibrium test $p$-value; ${ }^{d}$ Odds ratio based on logistic regression; ${ }^{\mathrm{e}} \mathrm{p}$-value

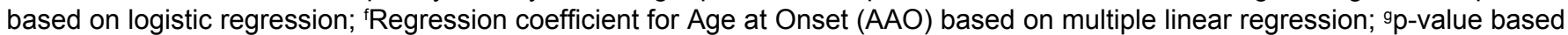
on linear regression.

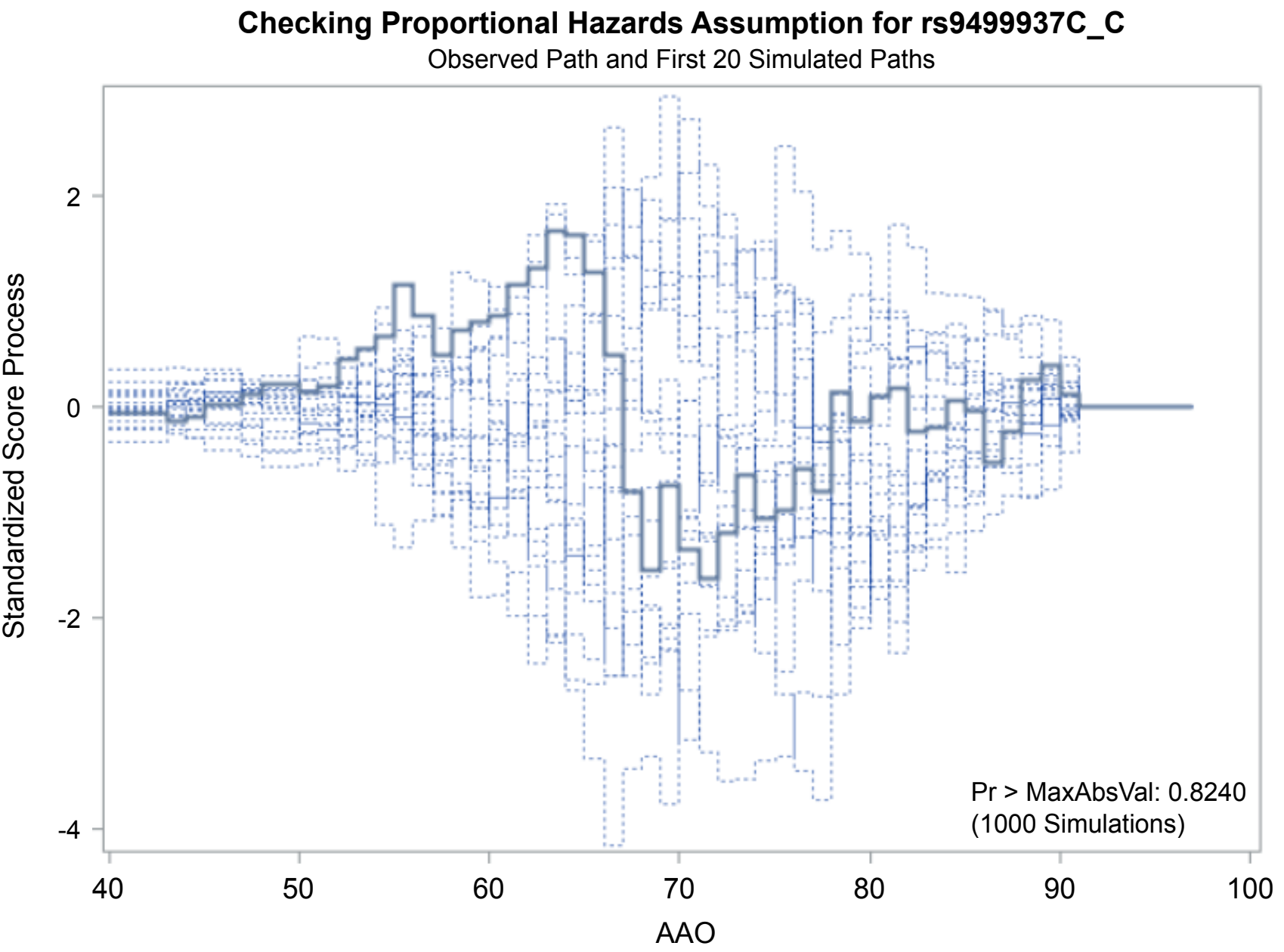

Figure 1: Explore plot for checking proportional hazards assumption for rs9499937C_C gentoype compared with rs9499937T_T genotype. 
Checking Proportional Hazards Assumption for rs9499937C_T

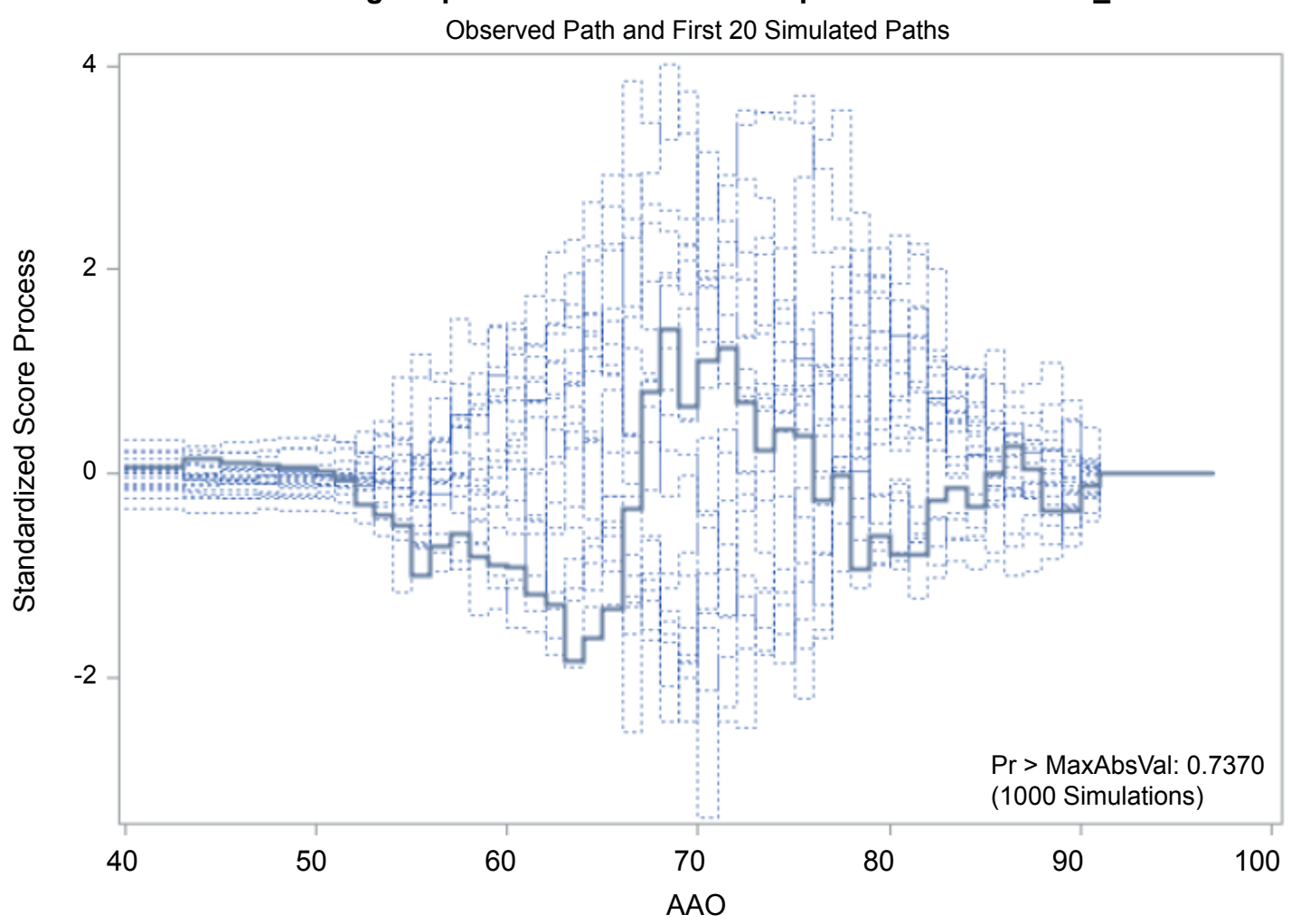

Figure 2: Explore plot for checking proportional hazards assumption for rs9499937C_T compared with rs9499937T_T.

Table 3: SNPs associated with the age at onset of AD using PROC PHREG $(p<0.05)$.

\begin{tabular}{|c|c|c|c|c|c|c|c|}
\hline SNP & $\mathbf{G T}^{\mathrm{a}}$ & $H^{b}$ & $p^{c}$ & $\mathrm{AlC}^{\mathrm{d}}$ & $H^{e}$ & HPD $^{f}$ & DIC $^{g}$ \\
\hline \multirow[t]{3}{*}{ rs7746856 } & $\mathrm{C}-\mathrm{C}$ & & & 7622.19 & & & 7622.21 \\
\hline & A-A & $1.28(1.04-1.57)$ & 0.0208 & & 1.28 & $1.02-1.56$ & \\
\hline & $A-G$ & $1.02(0.87-1.20)$ & 0.82 & & 1.02 & $0.86-1.19$ & \\
\hline \multirow[t]{3}{*}{ rs6941988 } & $\mathrm{T}-\mathrm{T}$ & & & 7622.24 & & & 7622.32 \\
\hline & $\mathrm{C}-\mathrm{C}$ & $1.27(1.03-1.56)$ & 0.026 & & 1.27 & $1.02-1.54$ & \\
\hline & C-T & $1.01(0.85-1.18)$ & 0.959 & & 1.01 & $0.85-1.18$ & \\
\hline \multirow[t]{3}{*}{ rs9499937 } & $\mathrm{C}-\mathrm{C}$ & & & 7616.16 & & & 7616.17 \\
\hline & C-T & $1.33(1.13-1.57)$ & $5.0 \times 10^{-4}$ & & 1.34 & $1.11-1.55$ & \\
\hline & T-T & $1.35(0.80-2.27)$ & 0.266 & & 1.35 & $0.68-2.06$ & \\
\hline \multirow[t]{3}{*}{ rs7770002 } & $A-G$ & & & 7622.37 & & & 7622.34 \\
\hline & $A-C$ & $1.27(1.03-1.57)$ & 0.0239 & & 1.28 & $1.02-1.55$ & \\
\hline & $\mathrm{C}-\mathrm{C}$ & $1.01(0.86-1.20)$ & 0.864 & & 1.02 & $0.85-1.19$ & \\
\hline
\end{tabular}

aTested genotype comparing with the reference; bHazards Ratio (HR) for the tested genotype based on classic Cox regression

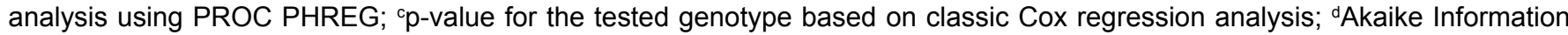
Criterion (AIC) value based on classic Cox regression analysis; eHR for the tested genotype based on Bayesian Cox regression analysis; fLower and upper 95\% Highest Posterior Density (HPD) of HR based on Bayesian Cox regression analysis; ${ }^{9}$ Deviance Information Criteria (DIC) value based on Bayesian Cox regression analysis.

analysis (Figure 3) indicated that the Markov chain had stabilized with good mixing for rs9499937. The posterior density plot, which estimates the posterior marginal distributions for the four regression coefficients, showed a smooth and unimodal shape for the posterior marginal distribution (Figure 4).

\section{Family-based association analysis}

We observed one SNP associated with the risk of AD ( $r s 9486018$ with $p=0.0323$ ) by using FBAT-GEE analysis in the family-based study. The T-A haplotype from rs6937432 and rs6940552 revealed mostly significant associations with the risk $\left(p=9.7 \times 10^{-4}\right)$. The T-T haplo- type from rs9786015 and rs9486018 and the T-G haplotype from rs9486018 and rs4079063 showed significant association with the risk $\left(p=1.56 \times 10^{-3}\right.$ and 4.46 $\times 10^{-3}$, respectively) (Table 4). Using the FBAT-Wilcoxon test, the C-C haplotype from rs6937026 and rs6946640 revealed mostly significant associations with the AAO $(p=0.0223)$. The C-A-G and C-C-A haplotype from rs6437026, rs4946640 and rs6910034 showed associations with the AAO ( $p=0.0274$ and 0.0225 , respectively). The T-T-G haplotype from rs9786015, rs9486018 and rs4079063 showed associations with both the risk $\left(p=2.27 \times 10^{-3}\right)$ and the AAO $(p=0.0487)$. 

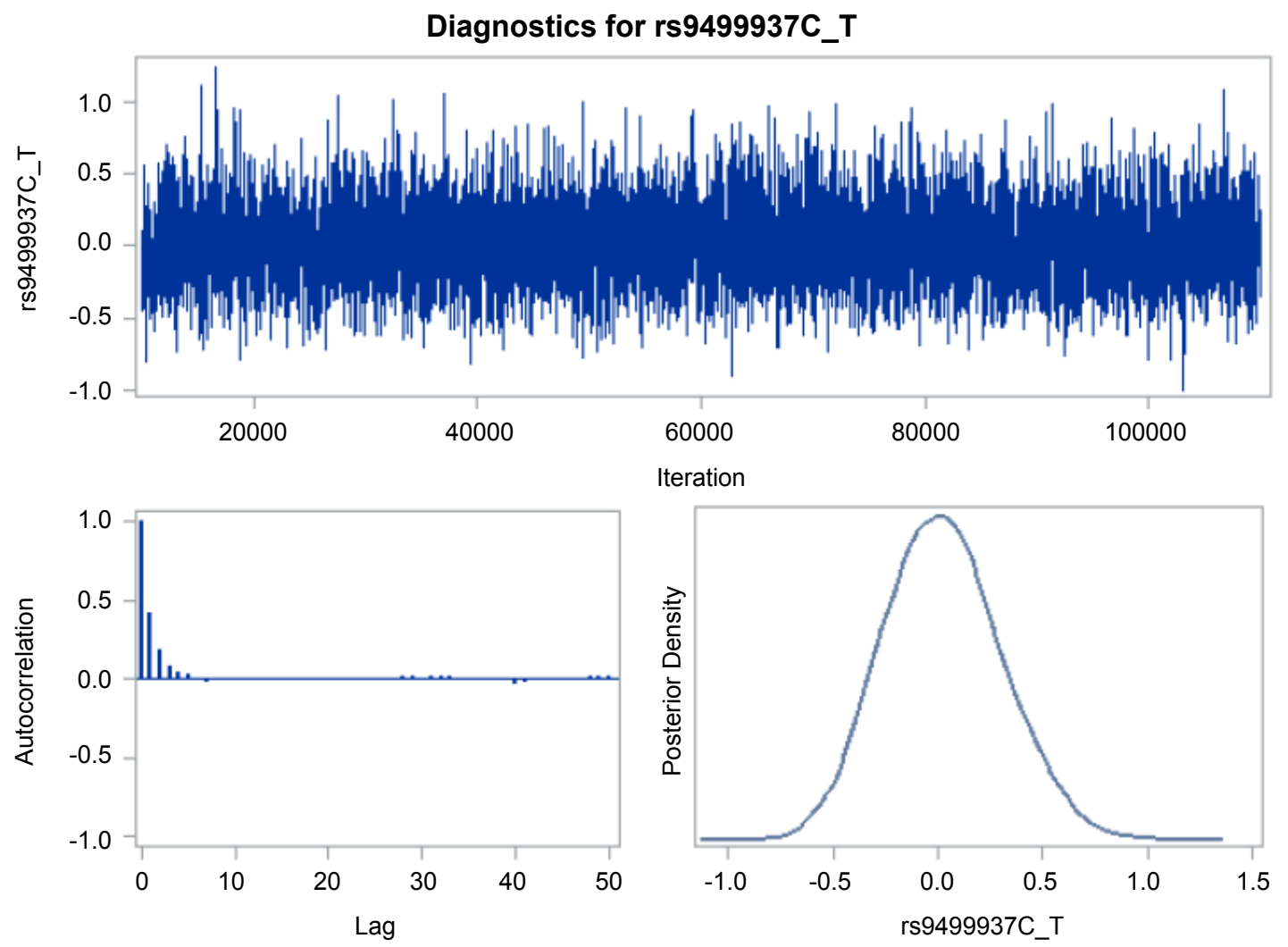

Figure 3: Trace plot, autocorrelation function plot, and posterior density plot for rs9499937.

Table 4: Haplotype analysis of the risk and age at onset of $A D$ in the family sample.

\begin{tabular}{|c|c|c|c|c|c|c|}
\hline & SNPs & & Haplotype $^{a}$ & Frequency $^{\mathrm{b}}$ & Fam\#c & p-value $^{d}$ \\
\hline \multicolumn{7}{|l|}{ Risk of $A D$} \\
\hline rs9499927 & rs9404573 & & $\mathrm{C}-\mathrm{C}$ & 0.01 & 37 & 0.00144 \\
\hline rs9404573 & rs9499934 & & C-T & 0.02 & 41 & 0.00355 \\
\hline rs6937432 & rs6940552 & & T-A & 0.01 & 37 & 0.00097 \\
\hline rs1378720 & rs13198196 & & C-T & 0.02 & 60 & 0.0147 \\
\hline rs9486015 & rs9486018 & & T-T & 0.06 & 101 & 0.00156 \\
\hline rs9486018 & rs4079063 & & T-G & 0.07 & 120 & 0.00446 \\
\hline rs9486015 & rs9486018 & rs4079063 & T-T-G & 0.04 & 54 & 0.00227 \\
\hline \multicolumn{7}{|c|}{ Age at onset of $A D$} \\
\hline rs6937026 & rs4946640 & & $\mathrm{C}-\mathrm{C}$ & 0.23 & 166 & 0.0223 \\
\hline \multirow[t]{2}{*}{ rs6937026 } & rs4946640 & rs6910034 & C-A-G & 0.51 & 156 & 0.0274 \\
\hline & & & C-C-A & 0.16 & 100 & 0.0225 \\
\hline \multirow[t]{2}{*}{ rs9486015 } & rs9486018 & rs4079063 & T-T-A & 0.01 & 22 & 0.0719 \\
\hline & & & T-T-G & 0.04 & 46 & 0.0487 \\
\hline
\end{tabular}

aHaplotype inferred from 2 or 3 SNPs; ${ }^{b}$ Haplotype frequency; ' ${ }^{\mathrm{F} A M \#}$ refers to the number of informative families using an additive model; ${ }^{d} p$-value for the haplotype based on FBAT-GEE analysis for the risk or based on FBAT-Wilcoxon analysis for the age at onset.

\section{The linkage disequilibrium structure of the HACE1} gene

Using the HapMap data, we identified one haplotype block including the four SNPs associated $A D$ and/or AAO in the case-control study. Figure 5 shows the LD structure based on LD statistics $\left(r^{2}\right)$. Based on the rough rule of thumb, values of $r^{2}>1 / 3$ might indicate sufficiently strong LD that can be used for a fine mapping [50]. The neuroblastoma associated rs4336470 [24,25] had moderate or strong LD with three AAO associated SNPs (rs7746856, rs6941988 and rs7770002 with $r^{2}=0.6,0.6$ and 0.71 , respectively) and weak LD with the risk and the AAO - asso- ciated SNP rs9499937 $\left(r^{2}=0.27\right)$. Furthermore, there was strong LD observed between rs2499663 and three AAO associated SNPs (rs7746856, rs6941988 and rs7770002 with $r^{2}=0.81,0.81$ and 0.93 , respectively). Using the founders in the family study, LD structure based on $\left(r^{2}\right)$ was constructed for all 28 SNPs (Figure 6). The two neuroblastoma associated SNPs (rs4336470 and rs9404576) $[24,25]$ had moderate LD with SNPs (such as rs6910034, rs9404573, rs9499934, rs696937432, rs9486015, and rs4079063) which built $A D$ and AAO associated haplotype; while another neuroblastoma associated SNPs rs2499663 [24,25] had moderate or strong LD with SNPs involved in $A D$ and $A A O$ associated haplotype. 

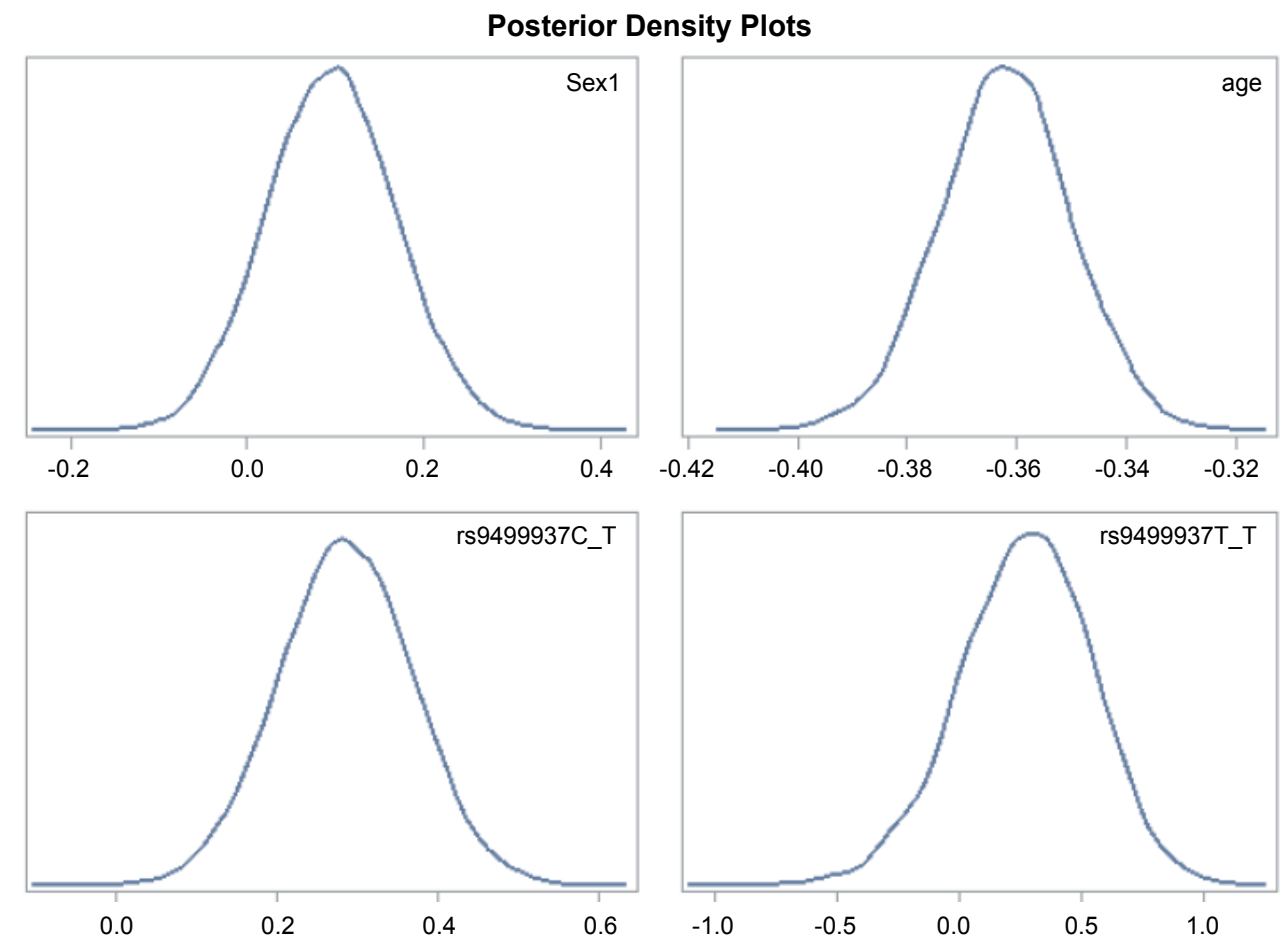

Figure 4: The posterior density plots for the 4 regression coefficients.

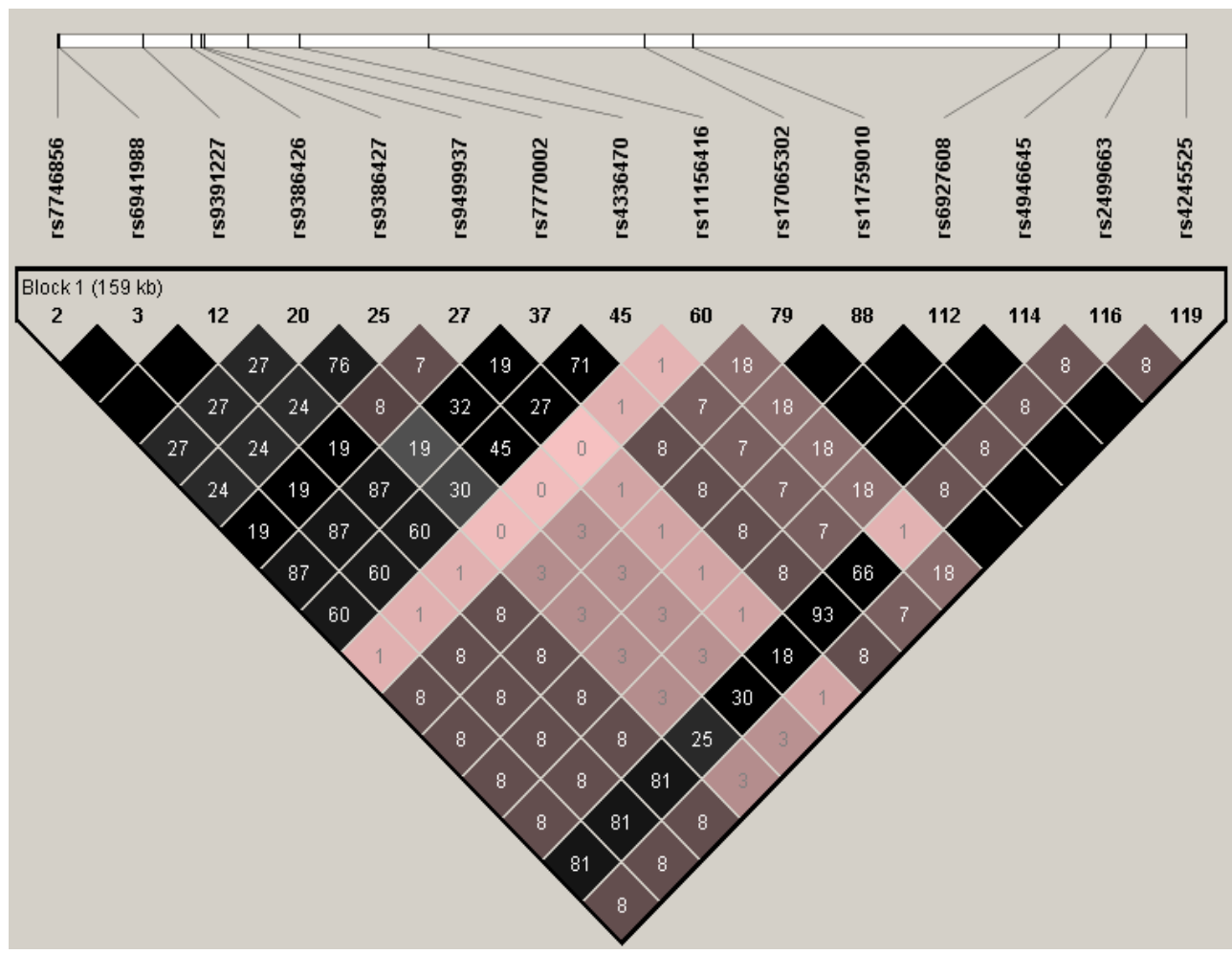

Figure 5: Linkage disequilibrium structure $\left(r^{2}\right)$ within the HACE1 gene using the HapMap data (Dark area shows $r^{2}=1$ ).

\section{Discussion}

In the present study we explored the association of 14 HACE1 SNPs with the risk and AAO of AD using a case-control study and identified one SNP associated with $A D$ and four SNPs with the AAO of $A D$ using PLINK software. Interestingly, the same SNP rs9499937 showed associations with both the risk and $A A O$ of $A D$. Bayesian Cox regressions revealed similar but a slight- ly stronger associations with the AAO of AD. Using an independent family-based sample, one SNP rs9486018 was associated with the risk of AD while haplotype analyses further revealed the associations with the risk and $A A O$ of $A D$. The findings of this study provide the first evidence that several genetic variants in the HACE1 gene influenced the risk and the $A A O$ of $A D$.

A previous study suggested that rs9391227 in the 


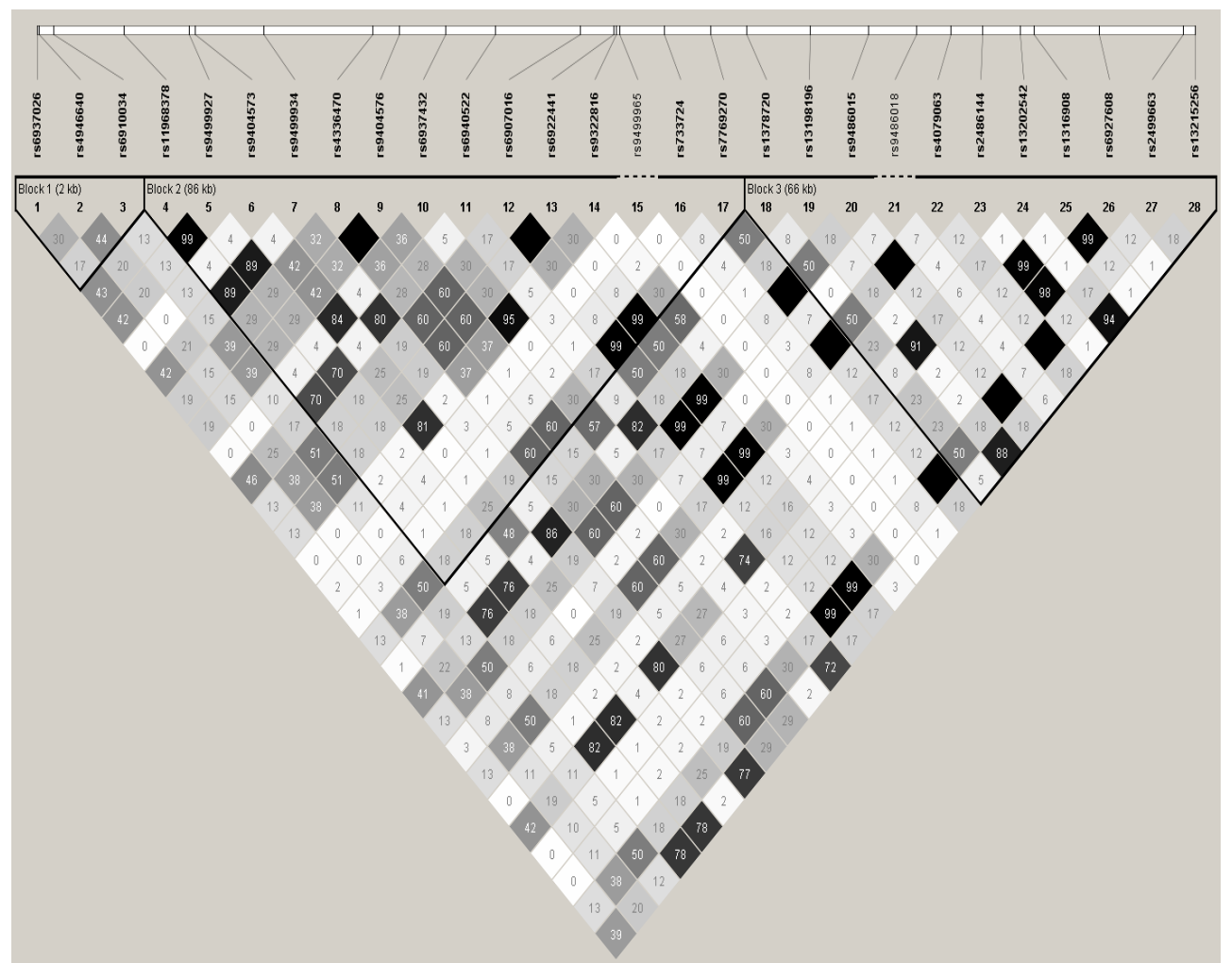

Figure 6: Linkage disequilibrium structure $\left(r^{2}\right)$ within the HACE1 gene of 28 SNPs in the family sample (Dark area shows $\left.r^{2}=1\right)$.

HACE1 gene was associated with celiac disease involved in the immune system and antigen presentation [51]; while another study revealed that five SNPs (rs17065302, rs11759010, rs6927608, rs4946645 and rs4245525) within HACE1 gene associated with equol-producing phenotype such as blood pressure [23] which may implicate HACE1 in immune responses. However, rs9391227 [51] was not available in both the case-control and family-based samples; while equol-producing phenotype associated five SNPs [23] were not associated with the risk or the $A A O$ of $A D$ in the case-control study and rs6927608 was not associated with the risk and AAO of $A D$ in both samples. However, rs9391227 had strong LD with three AAO associated SNPs (rs7746856, rs6941988 and $r 57770002$ with $r^{2}=1.0,1.0$ and 0.87 , respectively) in the case-control study (Figure 5); whereas rs6927608 had weak LD with other SNPs (Figure 5 and Figure 6). Previous studies have shown that AD is a chronic neurodegenerative disease while autophagy, immune and inflammatory processes are involved in the pathogenesis of $A D[1,8,9,52-56]$.

Previous epidemiology studies have suggested comorbidity of AD with certain cancers [57-59]; whereas there may be an inverse link between cancer and $A D$ [59-61]. Recently, a meta-analysis of nine studies supports an association between AD and decreased cancer risk [62]. However, the mechanism still remains unclear [60]. Several SNPs (such as rs4336470, rs9404576, rs4079063, rs24996663, and rs2499667) in the HACE1 have been found to be associated with neuroblastoma susceptibility [24-26]. In the present study, the T-G haplotype inferred from the rs9486018 and rs4079063 was found to be associated with the risk of $A D$; while the T-T-A haplotype inferred from the rs9486015, rs9486018 and rs4079063 was found to be associated with the $A A O$ of $A D$ in the family-based study (Table 4). Furthermore, four neuroblastoma associated SNPs (rs4336470, rs9404576, rs4079063 and rs2499663) [2426] had moderate to strong LDs with SNPs which built $A D$ and $A A O$ associated haplotypes (Figure 6). In addition, recently, it has been reported that HACE1 may play a role in neurodevelopment and addiction [19,21,22]. Taken together, the above findings may suggest that HACE1 gene may be involved in the pathogenesis of AD, cancers and blood pressure; however, the mechanism warrants further studies.

Several strengths of this study are worthy of noting. The present study provides the first evidence of several genetic variants within the HACE1 gene associated with the risk and the AAO of AD using a case-control sample and a family-based sample for replication. Furthermore, we checked the proportional hazards assumption using both the graphical and numerical methods for the Cox proportional hazards model and found that the proportional hazards assumption was valid for the AAO of $A D$ data in the Canadian sample. In addition, we conducted Bayesian survival analysis of genetic variants with the AAO of AD. Bayesian method may provide an alternative approach to assessing and verifying associations that alleviates the limitations of $p$-values at the cost of some additional modeling and it has recently made great inroads in genetic association studies [30]. Like other research studies, some limitations also exist in this study. First, due to different genotyping platforms, only 
two SNPs (rs6927608 and rs24999663) overlap in two samples; therefore, our replication results in the family-based sample are gene-based rather than SNP-based. Second, only one SNP rs9499937 was associated with the risk and $A A O$ of $A D$ in the case-control study; while one haplotype T-T-G was associated with the risk and $A A O$ of $A D$ in the family-based study; which revealed heterogeneity between risk and AAO of AD. In addition, our current findings might be subject to type I error and need to be replicated in future studies.

\section{Conclusion}

To the best of our knowledge, this is the first candidate gene study which investigated the associations of HACE1 SNPS with the risk of and the AAO of AD. The findings may serve as a resource for replication in other populations for future investigations on target genetic variation and $A D$. Future functional studies of this gene may help better characterize the genetic architecture of the risk of and $A A O$ of $A D$.

\section{Acknowledgments}

We acknowledge the NIH GWAS Data Repository, the Contributing Investigator(s) who contributed the phenotype data and DNA samples from his/her original study and the primary funding organization that supported the contributing study "Multi-Site Collaborative Study for Genotype-Phenotype Associations in Alzheimer's disease and longitudinal follow-up of Genotype-Phenotype Associations in Alzheimer's disease and Neuroimaging component of Genotype-Phenotype Associations in Alzheimer's disease" and "National Institute on Aging - Late Onset Alzheimer's Disease Family Study: Genome-Wide Association Study for Susceptibility Loci". The genotypic and associated phenotypic data used in the study, "Multi-Site Collaborative Study for Genotype-Phenotype Associations in Alzheimer's Disease (GenADA)" were provided by the GlaxoSmithKline, R\&D Limited. The datasets used for analyses described in this manuscript were obtained from dbGaP at https://www. ncbi.nlm.nih.gov/gap through dbGaP accession number phs000219.v1.p1. Funding support for the "Genetic Consortium for Late Onset Alzheimer's Disease" was provided through the Division of Neuroscience, NIA. The Genetic Consortium for Late Onset Alzheimer's Disease includes a genome-wide association study funded as part of the Division of Neuroscience, NIA. Assistance with phenotype harmonization and genotype cleaning, as well as with general study coordination, was provided by Genetic Consortium for Late Onset Alzheimer's Disease. The datasets used for analyses described in this manuscript were obtained from dbGaP at

https://www.ncbi.nlm.nih.gov/projects/gap/cgibin/study.cgi?study_id=phs000168.v1.p1.

\section{Role of the Funding Sources}

No founding source is given for the present paper.

\section{Conflict of Interest}

The authors declare that they have no conflict of interest.

\section{References}

1. Bird TD (2008) Genetic aspects of Alzheimer disease. Genet Med 10: 231-239.

2. Hebert LE, Weuve J, Scherr PA, Evans DL (2013) Alzheimer disease in the United States (2010-2050) estimated using the 2010 census. Neurology 80: 1778-1783.

3. Weuve J, Hebert LE, Scherr PA, Evans DA (2015) Prevalence of Alzheimer disease in US states. Epidemiology 26: e4-e6.

4. Beydoun MA, Beydoun HA, Gamaldo AA, Rostant OS, Dore GA, et al. (2015) Nationwide Inpatient Prevalence, Predictors, and Outcomes of Alzheimer's Disease among Older Adults in the United States, 2002-2012. J Alzheimers Dis 48: 361-375.

5. Steenland K, Goldstein FC, Levey A, Wharton W (2016) A Meta-Analysis of Alzheimer's Disease Incidence and Prevalence Comparing African-Americans and Caucasians. J Alzheimers Dis 50: 71-76.

6. Brookmeyer R, Johnson E, Ziegler-Graham K, Arrighi HM (2007) Forecasting the global burden of Alzheimer's disease. Alzheimers Dement 3: 186-191.

7. Niu H, Álvarez-Álvarez I, Guillén-Grima F, Aguinaga-Ontoso I (2017) Prevalence and incidence of Alzheimer's disease in Europe: A meta-analysis. Neurologia 32: 523-532.

8. Chesser AS, Pritchard SM, Johnson GV (2013) Tau clearance mechanisms and their possible role in the pathogenesis of Alzheimer disease. Front Neurol 4: 122.

9. Shin JY, Park HJ, Kim HN, Oh SH, Bae JS, et al. (2014) Mesenchymal stem cells enhance autophagy and increase $\beta$-amyloid clearance in Alzheimer disease models. Autophagy 10: 32-44.

10. Gatz M, Reynolds CA, Fratiglioni L, Johansson B, Mortimer JA, et al. (2006) Role of Genes and Environments for Explaining Alzheimer Disease. Arch Gen Psychiatry 63: 168-174.

11. Daw EW, Payami H, Nemens EJ, Nochlin D, Bird TD, et al. (2000) The number of trait loci in late-onset Alzheimer disease. Am J Hum Genet 66: 196-204.

12. Li YJ, Scott WK, Hedges DJ, Zhang F, Gaskell PC, et al. (2002) Age at onset in two common neurodegenerative diseases is genetically controlled. Am J Hum Genet 70: 985-993.

13. Nagase $T$, Kikuno R, Ishikawa K, Hirosawa M, Ohara $O$ (2000) Prediction of the coding sequences of unidentified human genes. XVI. The complete sequences of 150 new cDNA clones from brain which code for large proteins in vitro. DNA Res 7: 65-73.

14. Fernandez CV, Lestou VS, Wildish J, Lee CL, Sorensen PH (2001) Detection of a novel $t(6 ; 15)(q 21 ; q 21)$ in a pediatric Wilms tumor. Cancer Genet Cytogenet 129: 165-167.

15. Anglesio MS, Evdokimova V, Melnyk N, Zhang L, Fernandez CV, et al. (2004) Differential expression of a novel ankyrin containing E3 ubiquitin-protein ligase, Hace1, in sporadic Wilms' tumor versus normal kidney. Hum Mol Genet 13: 2061-2074.

16. Zhang L, Anglesio MS, O'Sullivan M, Zhang F, Yang G, et al. (2007) The E3 ligase HACE1 is a critical chromosome $6 q 21$ tumor suppressor involved in multiple cancers. Nat Med 13: 1060-1069. 
17. Küçük $C, \mathrm{Hu} X$, lqbal J, Gaulard $P$, Klinkebiel $D$, et al (2013) HACE1 is a tumor suppressor gene candidate in natural killer cell neoplasms. Am J Pathol 182: 49-55.

18. Gao ZF, Wu YN, Bai ZT, Zhang L, Zhou Q, et al. (2016) Tumor-suppressive role of HACE1 in hepatocellular carcinoma and its clinical significance. Oncol Rep 36: 3427-3435.

19. Rotblat B, Southwell AL, Ehrnhoefer DE, Skotte NH, Metzler M, et al. (2014) HACE1 reduces oxidative stress and mutant Huntingtin toxicity by promoting the NRF2 response. Proc Natl Acad Sci U S A 111: 3032-3037.

20. Zhang L, Chen X, Sharma P, Moon M, Sheftel AD, et al. (2014) HACE1-dependent protein degradation provides cardiac protection in response to haemodynamic stress. Nat Commun 5: 3430.

21. Hollstein R, Parry DA, Nalbach L, Logan CV, Strom TM, et al. (2015) HACE1 deficiency causes an autosomal recessive neurodevelopmental syndrome. J Med Genet 52: 797-803.

22. Cetinbas N, Daugaard M, Mullen AR, Hajee S, Rotblat B, et al. (2015) DeBerardinis RJ, Sorensen PH. Loss of the tumor suppressor Hace1 leads to ROS-dependent glutamine addiction. Oncogene 34: 4005-4010.

23. Hong KW, Ko KP, Ahn Y, Kim CS, Park SJ, et al. (2012) Epidemiological profiles between equol producers and nonproducers: a genomewide association study of the equol-producing phenotype. Genes Nutr 7: 567-574.

24. Diskin SJ, Capasso M, Schnepp RW, Cole KA, Attiyeh EF, et al. (2012) Common variation at 6q16 within HACE1 and LIN28B influence susceptibility to neuroblastoma. Nat Genet $44: 1126-1130$.

25. Capasso M, Diskin SJ, Totaro F, Longo L, De Mariano M, et al. (2013) Replication of GWAS-identified neuroblastoma risk loci strengthens the role of BARD1 and affirms the cumulative effect of genetic variations on disease susceptibility. Carcinogenesis 34: 605-611.

26. Zhang Z, Zhang R, Zhu J, Wang F, Yang T, et al. (2017) Common variations within HACE1 gene and neuroblastoma susceptibility in a Southern Chinese population. Onco Targets Ther 10: 703-709.

27. Li H, Wetten S, Li L, St Jean PL, Upmanyu R, et al. (2008) Candidate single-nucleotide polymorphisms from a genomewide association study of Alzheimer disease. Arch Neurol 65: 45-53.

28. Janicki SC, Park N, Cheng $\mathrm{R}$, Clark LN, Lee JH, et al (2014) Estrogen receptor $\alpha$ variants affect age at onset of Alzheimer's disease in a multiethnic female cohort. Dement Geriatr Cogn Disord 38: 200-213.

29. Wang KS, Liu X, Wang L, Briones D, Xu C (2014) Genetic variants in the SORL1 gene are associated with age at onset of Alzheimer disease: A survival analysis. International Journal of Medical Genetics 2014: 689832.

30. Stephens M, Balding DJ (2009) Bayesian statistical methods for genetic association studies. Nat Rev Genet 10: 681-690.

31. Sullivan SG, Greenland S (2013) Bayesian regression in SAS software. Int J Epidemiol 42: 308-317.

32. Stokes M, Chen F, Gunes F (2014) An introduction to Bayesian analysis with SAS/STAT $®$ software. Proceedings of the SAS Global Forum 2014 Conference, SAS Institute Inc, Cary, NC

33. Filippini N, Rao A, Wetten S, Gibson RA, Borrie M, et al. (2009) Anatomically-distinct genetic associations of APOE epsilon4 allele load with regional cortical atrophy in Alzheimer's disease. Neuroimage 44: 724-728.
34. Lee JH, Cheng R, Graff-Radford N, Foroud T, Mayeux R (2008) Analyses of the National Institute on Aging Late-Onset Alzheimer's Disease Family Study: implication of additional loci. Arch Neurol 65: 1518-1526.

35. Wang KS, Liu X, Xie C, Liu Y, Xu C (2016) Non-parametric survival analysis of EPG5 gene with age at onset of Alzheimer's disease. J Mol Neurosci 60: 436-444.

36. Barrett JC, Fry B, Maller J, Daly MJ (2005) Haploview: Analysis and visualization of LD and haplotype maps. Bioinformatics 21: 263-265.

37. Purcell S, Neale B, Todd-Brown K, Thomas L, Ferreira MA, et al. (2007) PLINK: A tool set for whole-genome association and population-based linkage analyses. Am J Hum Genet 81: 559-575.

38. Dunn OJ (1961) Multiple Comparisons Among Means. Journal of the American Statistical Association 56: 52-64.

39. Cox DR (1972) Regression models and life-tables. Journal of the Royal Statistical Society 34: 187-220.

40. Cantor AB (2007) SAS Survival Analysis Techniques for Medical Research. ( $3^{\text {rd }}$ edn), Cary, SAS Institute INC.

41. George B, Seals S, Aban I (2014) Survival analysis and regression models. J Nucl Cardiol 21: 686-694.

42. Kasza J, Wraith D, Lamb K, Wolfe R (2014) Survival analysis of time-to-event data in respiratory health research studies. Respirology 19: 483-492.

43. Lin D, Wei LJ, Ying Z (1993) Checking the Cox Model with Cumulative Sums of Martingale-Based Residuals. Biometrika 80: 557-572.

44. Akaike H (1979) A Bayesian Extension of the Minimum AIC Procedure of Autoregressive Model Fitting. Biometrika 66: 237-242.

45. Akaike H (1981) Likelihood of a Model and Information Criteria. Journal of Econometrics 16: 3-14.

46. Spiegelhalter DJ, Best NG, Carlin BP, Van der Linde A (2002) Bayesian Measures of Model Complexity and Fit (with Discussion). J R Statist Soc B 64: 583-639.

47. Van Steen K, Lange C (2005) PBAT: A comprehensive software package for genome-wide association analysis of complex family-based studies. Hum Genomics 2: 67-69.

48. Lange C, Silverman EK, Xu X, Weiss ST, Laird NM (2003) A multivariate family-based association test using generalized estimating equations: FBAT-GEE. Biostatistics 4: 195-206.

49. Lange C, Blacker D, Laird NM (2004) Family-based association tests for survival and times-to-onset analysis. Stat Med 23: 179-189.

50. Ardlie KG, Kruglyak L, Seielstad M (2002) Patterns of linkage disequilibrium in the human genome. Nat Rev Genet 3: 299-309.

51. Einarsdottir E, Bevova MR, Zhernakova A, Monsuur A, Koskinen LL, et al. (2011) Multiple independent variants in 6q21-22 associated with susceptibility to celiac disease in the Dutch, Finnish and Hungarian populations. Eur J Hum Genet 19: 682-686.

52. Holmes C, Cunningham C, Zotova E, Woolford J, Dean C, et al. (2009) Systemic inflammation and disease progression in Alzheimer disease. Neurology 73: 768-774.

53. Takeda S, Sato N, Morishita R (2014) Systemic inflammation, blood-brain barrier vulnerability and cognitive/ non-cognitive symptoms in Alzheimer disease: relevance to pathogenesis and therapy. Front Aging Neurosci 6: 171. 
54. Blach-Olszewska Z, Zaczynska E, Gustaw-Rothenberg K, Avila-Rodrigues M, Barreto GE, et al. (2015) The Innate Immunity in Alzheimer Disease- Relevance to Pathogenesis and Therapy. Curr Pharm Des 21: 3582-3588.

55. Heppner FL, Ransohoff RM, Becher B (2015) Immune attack: the role of inflammation in Alzheimer disease. Nat Rev Neurosci 16: 358-372.

56. López González I, Garcia-Esparcia P, Llorens F, Ferrer I (2016) Genetic and Transcriptomic Profiles of Inflammation in Neurodegenerative Diseases: Alzheimer, Parkinson, Creutzfeldt-Jakob and Tauopathies. Int J Mol Sci 17: 206.

57. Burke WJ, McLaughlin JR, Chung HD, Gillespie KN, Grossberg GT, et al. (1994) Occurrence of cancer in Alzheimer and elderly control patients: an epidemiologic necropsy study. Alzheimer Dis Assoc Disord 8: 22-28.
58. Roe CM, Behrens MI, Xiong C, Miller JP, Morris JC (2005) Alzheimer disease and cancer. Neurology 64: 895-898.

59. Roe CM, Fitzpatrick AL, Xiong C, Sieh W, Kuller L, et al. (2010) Cancer linked to Alzheimer disease but not vascular dementia. Neurology 74: 106-112.

60. Thinnes FP (2012) Why cancer survivors have a lower risk of Alzheimer disease. Mol Genet Metab 107: 630-631.

61. Musicco M, Adorni F, Di Santo S, Prinelli F, Pettenati C, et al. (2013) Inverse occurrence of cancer and Alzheimer disease: A population-based incidence study. Neurology 81: 322-328

62. Shi HB, Tang B, Liu YW, Wang XF, Chen GJ (2015) Alzheimer disease and cancer risk: a meta-analysis. J Cancer Res Clin Oncol 141: 485-494. 